\title{
Pattern Recognition of Thermal Images for Monitoring of Breathing Function
}

\author{
Fida' Al-Obaisi ${ }^{1}$, Ja'far Alqatawna ${ }^{2}$, Hossam Faris ${ }^{2}$, Ali Rodan ${ }^{2}$ and Omar Al- \\ $\mathrm{Kadi}^{2}$ \\ ${ }^{1}$ The Department of Engineering and Mathematics, Sheffield Hallam University, \\ $U K$ \\ ${ }^{2}$ King Abdulla II School for Information Technology, The University of Jordan, \\ Amman, Jordan
}

\begin{abstract}
Breathing is a crucial human process that requires easy and accurate monitoring. There are several available breathing monitoring methods which are based on strapping subjects with special sensors for monitoring their breathing rate. The results of these methods have been acceptable but connecting people with such equipment is awkward, especially in the case of monitoring the breathing of infants who have tiny and sensitive bodies. Therefore, providing a noncontact method is a good alternative. Thermal imaging is one of the latest non-contact passive techniques that are used for monitoring and controlling different human processes, including breathing. In this paper, a thermal image feature extraction method with neural network based classification system is developed to be used as part of a breathing monitoring system. The classification is based on matching the entries of mixed breathing images, either breath-in or breath-out images, with their desired values. The extracted breathing image features are used as entries for the neural network classifier. The neural network based model has produced promising classification results. Additionally, it has been possible to calculate the breathing rate of each subject from the classification results.
\end{abstract}

Keywords: Thermal image, Pattern recognition, Neural network, Breath monitoring

\section{Introduction}

Breathing is the bodily process of respiration, during which air moves in and out of the lungs. The body takes oxygen $\left(\mathrm{O}_{2}\right)$ from inhaled air and releases carbon dioxide $\left(\mathrm{CO}_{2}\right)$ by exhalation. Breathing plays a critical role in the diagnosis and control of respiratory diseases especially for new babies who are born with breathing problems. The number of breath count per interval time is called the breathing rate and it has a range of 12-18 cycles per minute for adults [1].

Monitoring of breathing function (or respiratory rate) has its applications in polygraphy, sport training, sleep tests, and patient monitoring [2]. The contact breathing applications were introduced as a first method for estimating respiratory rate. These techniques were based on the changes in electrical impedance pneumography signal across the chest, electrocardiogram, or the changes in light absorption across the finger [3]. Electrocardiogram (ECG) has been developed by attaching special sensors to the subject. The ECG principle based on the fact that the heart rates of the subjects are typically modulated by breathing. The signal corresponding to the heart function contains breath information. A number of studies have developed an improved technique of the ECG method called BioMatt [4]. This technique performs measurement of vital signs and cardiac activity without electrodes. Some researchers have developed a signal processing technique to separate out BioMatt components, because it was not possible to distinguish motion due to the breathing versus cardiac activity or body track [5]. The other option of 
the ECG method is Photoplethysmography (PPG). PPG is based on measuring blood volume changes in living tissues by absorption or scattering near infrared radiation [3].

Nevertheless, using heart function as a measurement basis to acquire the breathing waveform is unreliable because sinus arrhythmia is not present in all individuals [2]. Another contact method implemented earlier was the nasal temperature probe. It is implemented by using a thermistor that measures nasal air temperature changes as an indicator of breathing. The subject's chest is strapped around by the abdominal strain gauge transducer and the measurement comes from the change in thoracic or abdominal circumference while breathing. These contact methods require a close contact to subject which is quite uncomfortable and awkward (like abdominal transducer) [6].

A non-contact, touch-less or contact free method is a more suitable technology used for monitoring breathing function instead of contact types. Authors in [1] argued that a novel non-contact breathing monitoring has potential benefits in monitoring respiratory diseases. The non-contact breath monitoring is more convenient for patients.

Greneker in [7] has reported that the first non-contact method developed by researchers at Georgia Tech Research Institute (GTRI). They implemented a system to monitor respiration heart beat without any physical connection to the subject, the system is called Radar Vital Signs Monitor (RVSM). RVSM is very sensitive to small body movements and those movements could corrupt the breathing signal so the particular filters need to be implemented to solve the problem [8]. Moreover, in the year 2000, Infrared imaging proved it's possible in deception detection when thermal image analysis was used to detect facial patterns of stress at distance. The infrared approach is based on the nose surface temperature changes during the breathing processes (breath in/ breath out). It can be used as a selective signature which can be captured by infrared image [2]. This signature is now available for monitoring any disease diagnoses and it will be helpful to be applied in respiratory rate monitoring systems. In the context of this study, this signature is captured using infrared thermal imaging in order to develop the breathing monitoring system. This raised the need for developing a system which will be able to classify the subjects breathing thermal images.

Although the thermal contact-less breathing monitoring technique represents a convenient method for monitoring breathing in infants, it is not straightforward as one could imagine. The captured thermal images required further processing in order to fulfill the requirements of the monitoring function. Among these requirements is the need for extracting the features, which represent the breathing signature, from the capture thermal image. Extracting these features will produce a Region of Interest (ROI) which needs to be converted into another suitable form that can be processed in the later steps. This also reduces the amount of the data that will be required for the remaining processes. Another essential requirement for the breathing monitoring system is the ability to classify the patterns of the ROI into the basic breathing cycles either breath in or breathe out. The accuracy of the classification process is very important for the success of the monitoring system. Accurate classification depends on a number of factors which include quality of the thermal image, the chosen features extraction method and the classification technique.

In this paper we will develop a neural network based classification system to be used in infant's breath monitoring system and determining breathing rate. The developed classification system will be evaluated based on recognition of breath in/breath out cycles from thermal images.

The paper has the following organization: Section 2 gives a background on Thermal imaging and Multi-Layer Perceptron (MLP) neural network. In Section 3 we propose a thermal image based breathing monitoring system. Experiments and Results are presented in Section 4. Finally, our work is concluded in Section 5. 


\section{Background}

\subsection{Thermal Imaging}

Thermal imaging is a visualisation of temperature differences by converting of radiated or reflected objects heat to real time images. Each object is able to emit radiation and it can be thermally imaged [9]. Thermal imaging uses Infrared technology to detect the object temperatures. Forward Looking InfraRed (FLIR) camera systems have a capability to convert each visual material infrared radiation to electrical signal which can be displayed visually on a screen and stored.

Thermography is the leading of non-intrusive, non-invasive and non-destructive tool which is employed in a variety of applications across a wide range of industries. It is involved in applications such as electrical, mechanical, medical, Research and development, and Aerial. Thermal imaging is used in medical studies to measure the thermal energy emitted from subjects. This emitted radiation has a long wavelength which is too long to be detected by human eye. Thermal cameras are able to detect heat from human body to aid in making diagnoses of a number of healthcare conditions. Thermal imaging was used before to detect a breast cancer and assess the pain. Frize and others analyzed the thermal images for breast and pain patients to detect the dysfunction of both conditions [10]. They have achieved promising results and further compared them with actual clinical outcomes by statistical analysis. The replacement of the conventional contact method by a developed non-contact approach (i.e. Thermal imaging) was shown to be effective in the field of polygraph and for monitoring breath rate [2]. The computed breathing function replaces the polygraph channel (e.g. abdominal transducer) via infrared images.

The first reported use of infrared imaging for monitoring facial patterns detection at a distance was by Pavlidis and others in [11]. In [12], Pavlidis and Levine reported that breathing have significant impact on applications such as sleep studies, and polygraph testing. In sleep studies, infrared imaging can measure the physiology of hemodynamic and the autonomic nervous system. For polygraph testing, the aim is to ascertain if the subject under investigation is truthfully or deceitfully answers depending on subject's physiological parameters such as blood volume, pulse change, respiratory changes, and electrodermal activity. Thermal Image understanding is a complex process and has many different disciplines. Many researchers are involved in solving sub-problems such as edge detection, feature extraction, and low level segmentation. A number of researchers developed a neural networks model which allows clustering and classification of analyzed images [13-14].

Authors in [15], extracted thermal image features by using a thermal image sensor device. This sensor has an array of eight sensing elements aligned vertically and is mechanically rotated horizontally when scanning. Their neural system is trained with features after a pre-processing stage then the image position is measured and corresponding posture prior to implementation. They implemented a Multi-Layer Perceptron (MLP) neural network to detect the posture and the position of occupant. Authors also made a small neural system that use low resolution thermal images which are obtained from non-cooled, small, lightweight thermal sensor.

Other works develop the method for monitoring the respiration rate thermal images relaying on extraction of an ROI around the area of tip of the noise [16-17]. Their method showed good results even during small various baby head movements; nevertheless, the reported method requires an efficient classification method to deal with the extracted features. 

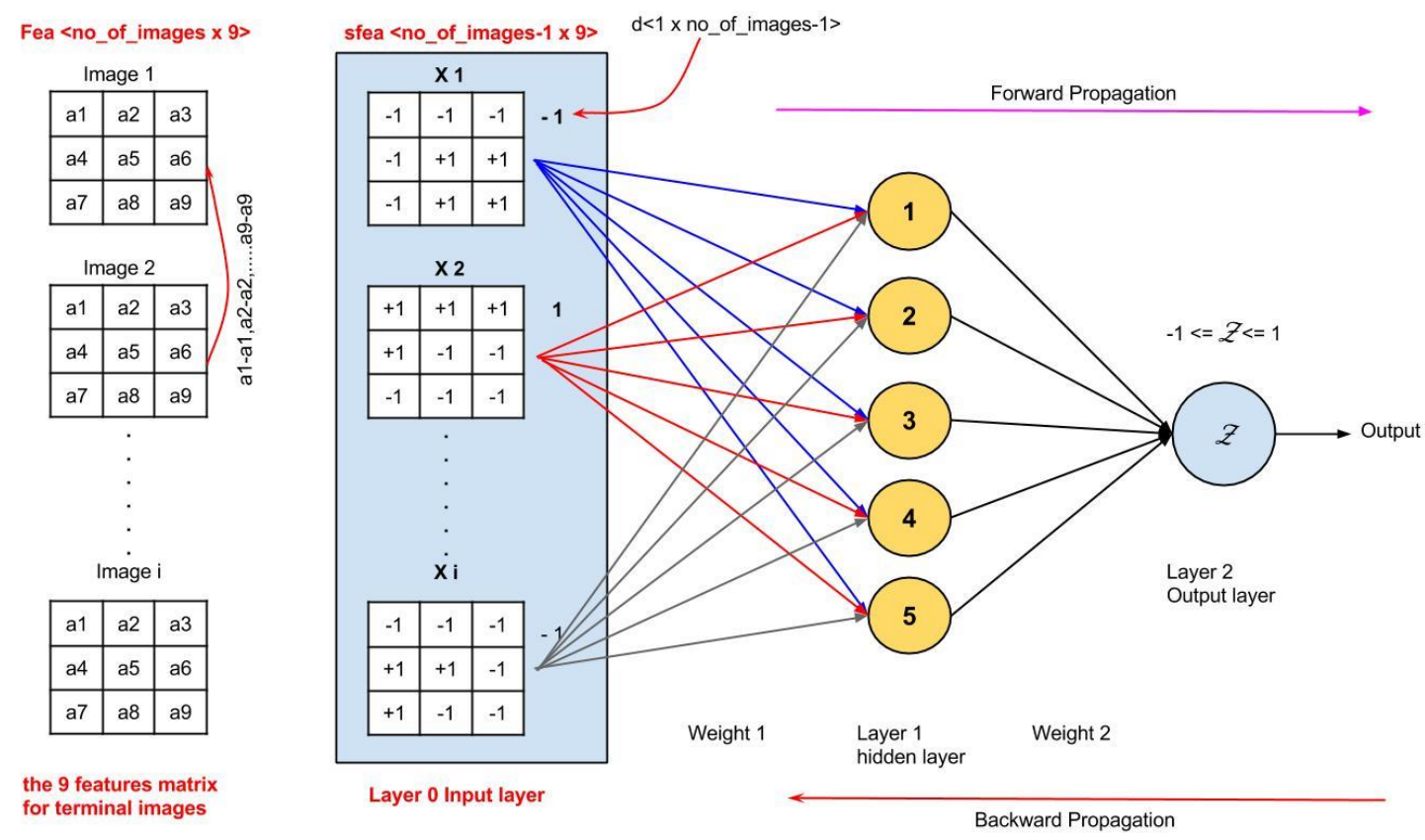

Figure 1. MLP Neural Network Classifier

\subsection{Multi-Layer Perceptron (MLP) Neural Network}

Artificial Neural Networks (ANNs) are computational models that are inspired by the human brain. ANNs are generally presented as a system of interconnected neurons (processing units) that computes the input stream. Each neuron has a number of input values which are processed by a summation function that multiplies the inputs and connection weights (modified inputs) and produces an output for the threshold value and activation function [18]. The use of different types of these neural networks depends on nature of applications.

Multi Layer Perceptron (MLP) is one of the most common types of ANN. MLP is mostly used for nonlinear pattern recognition which has the capability to solve some difficult and diverse problems by training them with the classical back propagation algorithm. The basic function of nonlinear separable patterns is carried out by adding a new layer called the hidden layer. Each input from the input layer is weighted and propagated through each node in hidden layer using an activation function to produce an output at the output layer. There are different types of activation functions, in this study we will use the hyperbolic tangent function which is defined by the following equation:

$$
\begin{gathered}
\tanh x=\frac{\sinh x}{\cosh x} \\
y=\varnothing(s)=\frac{1-e^{-s}}{1+e^{-s}}
\end{gathered}
$$

The hyperbolic is an odd function and it is symmetric around the origin, where its desired value is either 1 or -1 depending on the value of $s$ in the activation function. If $s>0$, then the output values are around 1 ; and if $s<0$, then the output values are around -1 because the output range is $-1 \leq y \leq 1$ [19]. Figure 1 describes the MLP classification system for this study. 
The training process in the MLP is carried out by forward and backward phases. In the forward phase, the error is calculated by minimizing any loss function. This error is used by the back propagation learning algorithm to adjust the weights. The output from each node is recalculated by using a new set of weights. Each time this process is repeated, the error is reduced. The training parameters used by MLP are:

1. Learning Rate: The learning is computed by the steepest descent method. This rate range is $0<h<1$. The smaller rate chosen gives a smaller weight connection and this gives a smoother learning step from one iteration to the next.

2. Momentum: A positive number used to control feedback loop acting around weight sets.

3. Number of iterations: The training epoch's (repeating) time.

4. Normalised SD: The MLP inputs are normalised to get zero mean and unity SD before training.

5. Gradient vector: This vector is used to optimize the weight values. In this study, we used two videos. The first one had 29 images and they were divided into; 20 images for training and 8 images for testing the neural system. The second video had 49 images; 35 images used for training and the other 14 images used for testing. After subtraction, the images were automatically reduced by one image. A number of images were ignored because of their unconfirmed status, whether in or out. The operation of choosing a number of images in each mode (test or train) is not fixed, but they can be placed randomly depending on the neural model and the size of data we have. \% In both videos, we tried many times to get the suitable number of images for both training and testing modes which provided the optimal classification results than the other choices.

\section{Proposed Breath Monitoring System}

Figure 2 shows an overall block diagram of the classification system design. The subjects only need to breath normally and the thermal camera converts each point in front of its lens into a corresponding thermal point. All recorded videos are stored and processed into A-40 camera software. Before classifying any breathing thermal images we need to ensure that the thermal image processing methods are implemented and tested through the first two steps of the software process. The extracted features with the desired image values were used in the neural network models as input patterns to get classify breathing (either breath in or breath out). Once the thermal image input patterns are prepared they are propagated through the MLP. The two available breathing videos for this study have a different number of images. The first has 29 images and the second has 49 images. They recorded the normal breathing processes of healthy people. Both videos are classified through MLP neural networks. The final results are shown in Tables 2 and 3, which display the image numbers, the calculated value (the output from the neural system), the class type (desired values in the neural network, -1 for breath in and 1 for breath out), and class name. It is possible to produce some mismatched or misclassified images, but the chances of this are small due to the data set size and the method of choosing a desired value. 


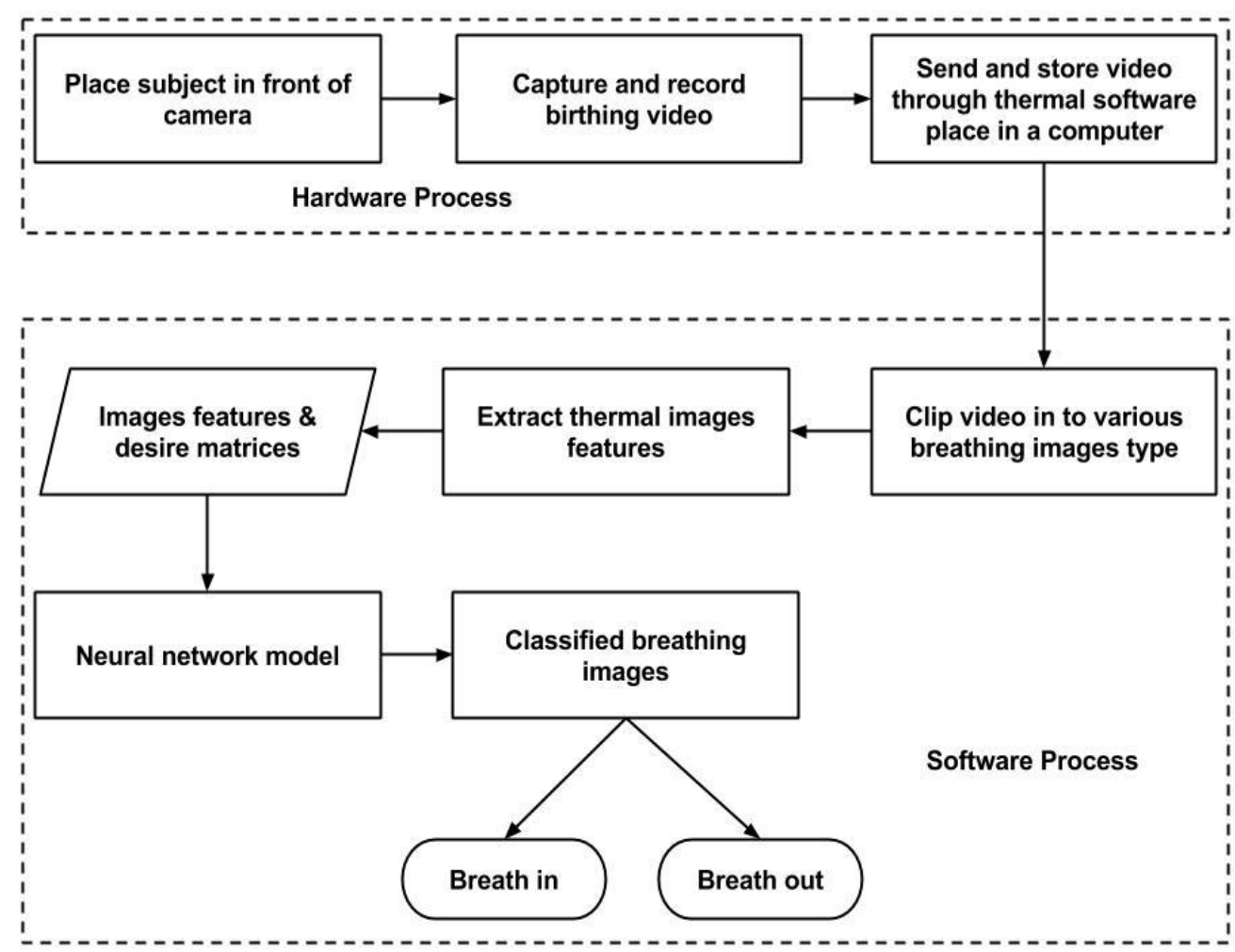

Figure 2. Example of Breathing Classification Results

\section{Experiments and Results}

\subsection{Experiments Setup and Features Extraction}

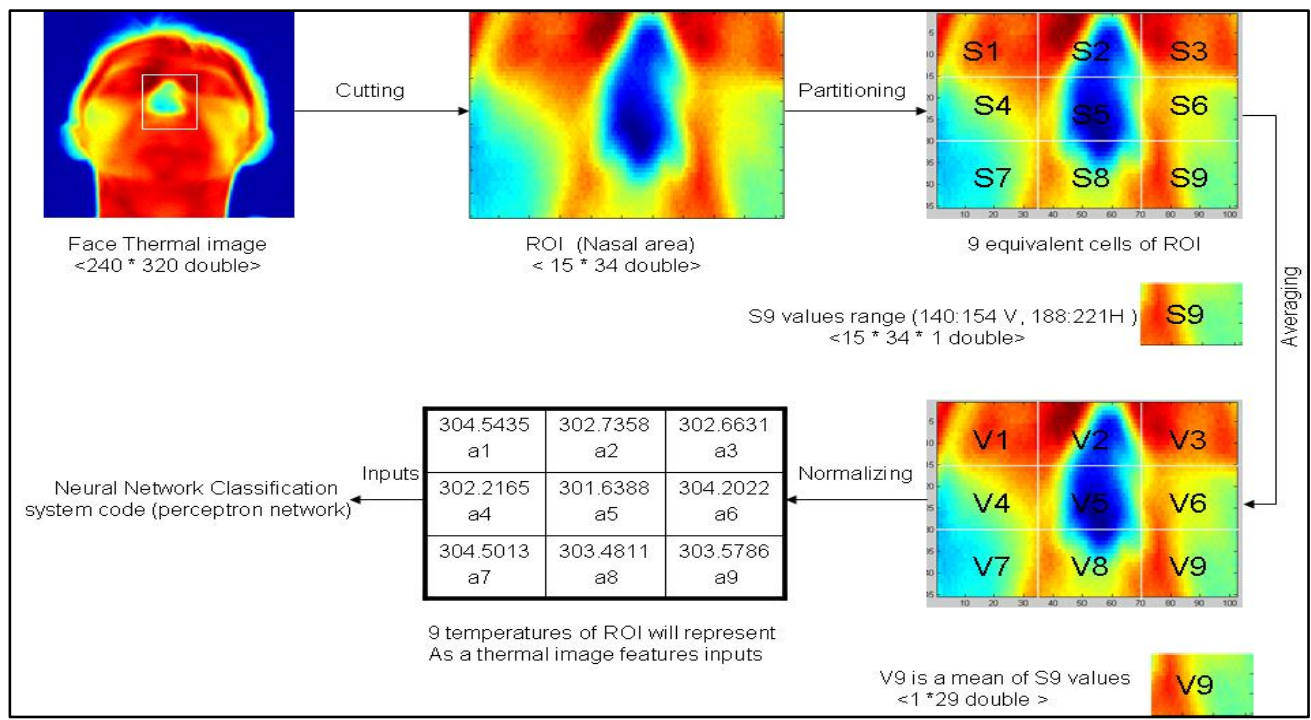

Figure 3. Thermal Image Feature Extraction Producers 
A FLIR A-40 thermal measurement infrared is connected to a laptop via a USB port. Once we connect the camera, and once the FLIR software (ThermaCAM ${ }^{\mathrm{TM}}$ Researcher pro 2.9) is running, the real time colour video automatically appears. After preparing the above system, the subject (the person who will be monitored) begins breathing normally once the video starts recording. Each second of the video produces an image. For this work, which recorded as much as possible, we got a huge number of breathing photos for each subject. Sometimes a few images can be ignored if they are not clear enough, especially for the nasal area. A clear image gives accurate features which helps the neural network model to classify properly. The FLIR A-40 camera captures 100 images at a time.

Up until now, we can distinguish that facial thermal image in Figure 3 is breath in/ breath out by observing the differences between the nasal area colors for each one, as well as from the average breathing plots in Figures 4 and 5. The breath out air coming from the nasal area is hotter than the breath in air. This can be easily shown by dragging the computer mouse through the image and the temperatures scale in front of the image shows the temperature for each pixel in that image. However, this is not a recommended method for classification due to the huge number of images that have to be monitored and classified.

After capturing and recording breathing videos, each image in the video is ready for cutting of the nasal area from whole image (the image here is a facial image only). Where the nasal area is our system's Region of Interest (ROI).

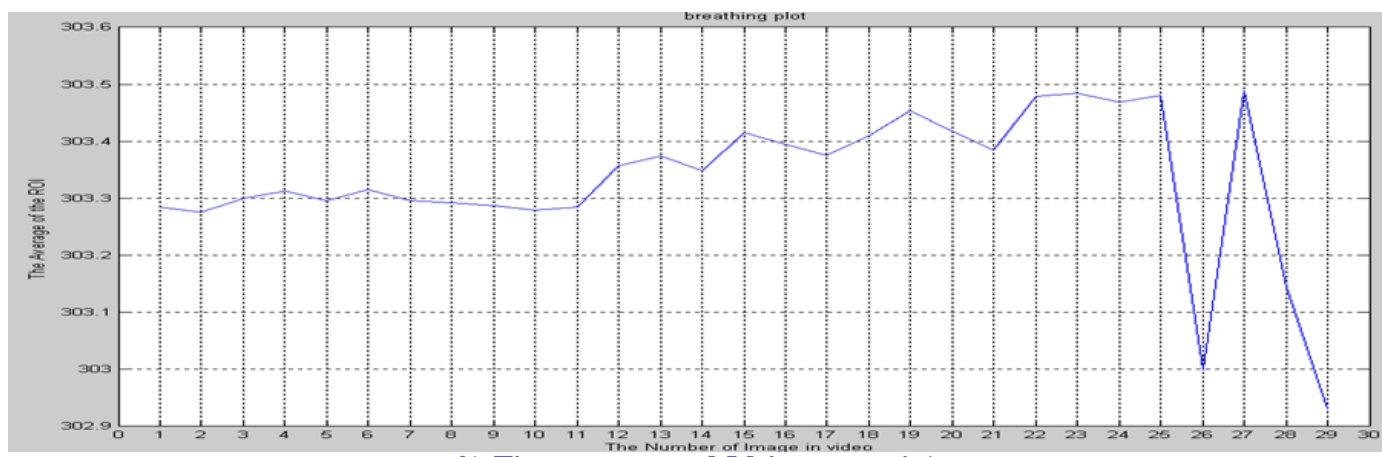

A) The average of 29 images plot

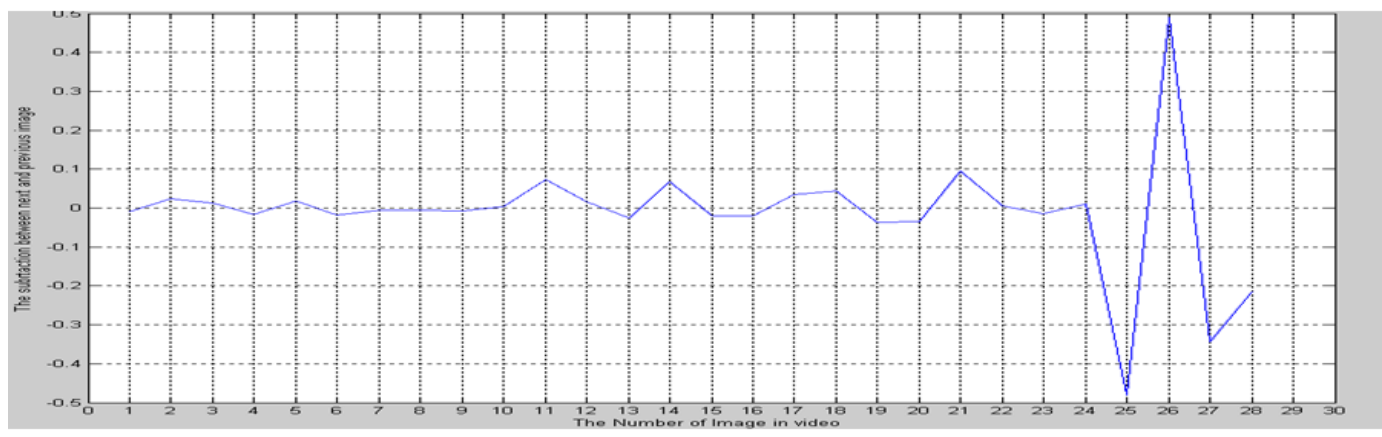

B) The subtraction average of 29 images plot

Figure 4. Average and Subtraction Plots for Short Video

Feature extraction is performed on the nasal area by dividing each ROI into nine equivalent cells. After that, the large number of pixels $(S)$ in each partition is reduced by averaging all the values into $(V)$ value partitions. These $(V \mathrm{~s})$ are averaged again and one value for each image cell is produced which is represented as one temperature $(a)$ in Figure 3. $\left(V_{0}\right)$ is given the (mean $S$ ) value for each $S$ in the image and these results are shown in part A of Figures 4 and 5. 
Figure 3 displays one image feature extraction producer. The steps are repeated for all images we have by involving them in code looping for production of nine features for each image. Note, the size of the partition (ROI, $S$, and $V$ ) matrices values may not have the same pictures sizes because they are scaled here for demonstrating purposes.

Instead of having hundreds of pixels in each image we have only nine values and they represent the thermal image in the classification system as a signature and inputs for the MLP.

Then, the neural network data are pre-processed and the features are extracted. In the neural network system, the subtraction is done between each image feature and the previous one. In this case, the gradients give more detail about the image sequences and the results will be more accurate depending on the image sign changes. This means that when the sign produced between 9 features subtraction from image 2 and image 1 becomes ( - ) value (Figures 4 and 5 part B), the desired value of the image is -1 "breath in" which means the image plot goes down and the temperature becomes cold; and if the sign is $(+)$ value, the desired value of the image is 1 "breath out" which means the image plot goes up and the temperature becomes hot. Part B in Figures 4 and 5 is used as a first desired value selection source, used for making the decision for each image process (either in or out).

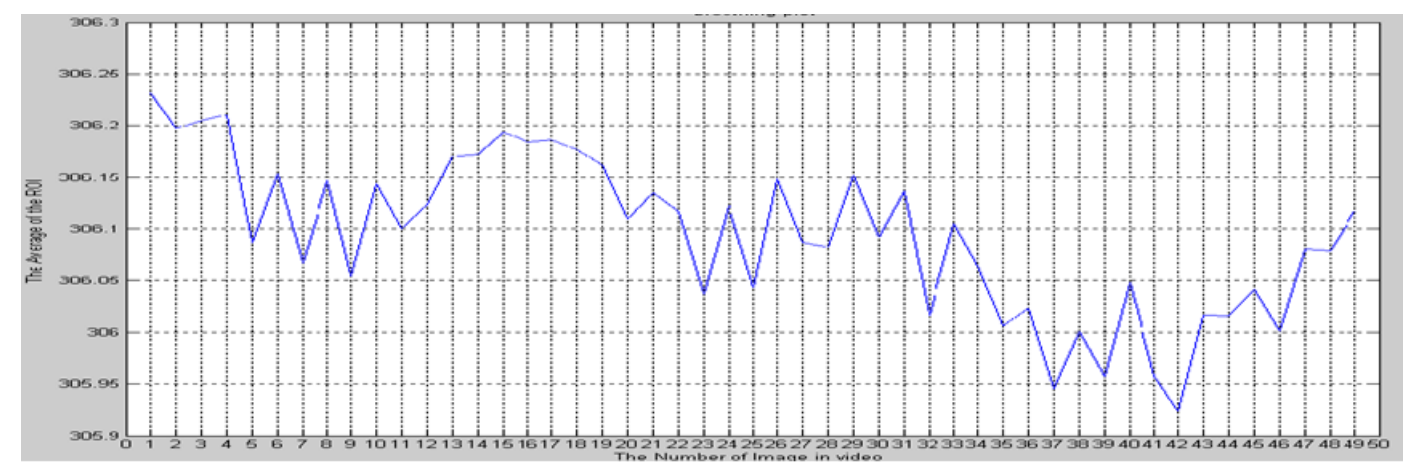

A) The average of 49 images plot

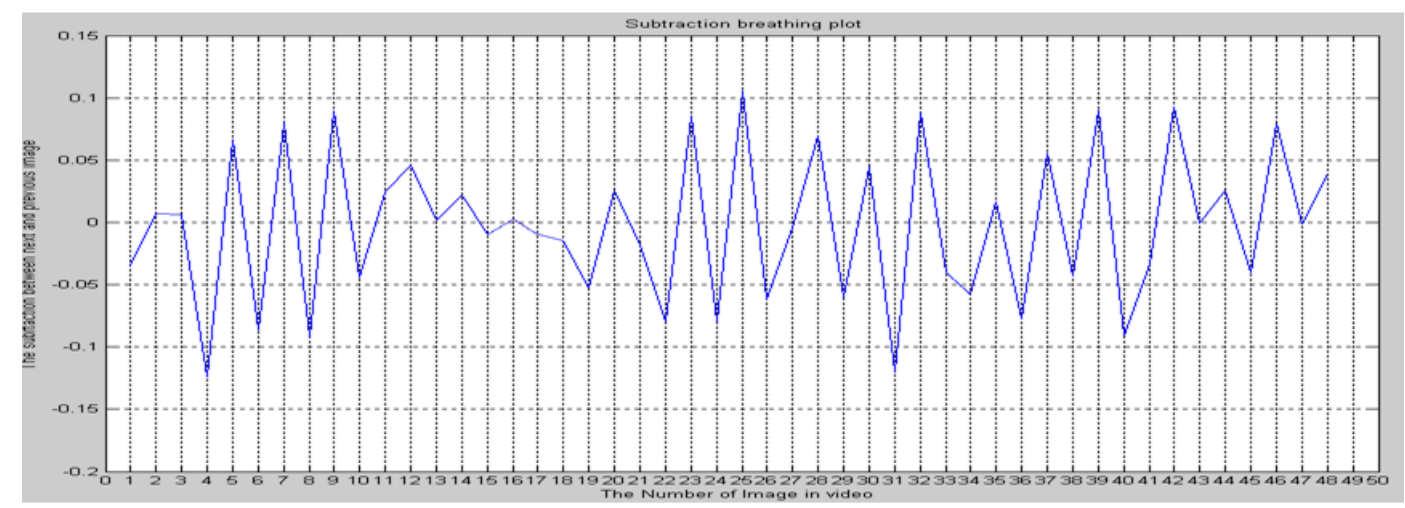

B) The subtraction average of 49 images plot

Figure 5. Average and Subtraction Plots for Long Video

\subsection{MLP Classification Results}

In our experiments, the training mode was based on minimizing the Root Mean Square Error (RMSE) function which is given in Equation 3.

$$
R M S E=\sqrt{\frac{\sum_{i=1}^{n}(y(i)-d(i))^{2}}{n}}
$$


The MLP parameters were tuned as shown in Table 1. Sometimes the training stopped at specific iterations once the RMSE converges to zero, meaning the system was well trained in that iteration value. Our videos did not reach iteration no. $=50000$, but the training in 29 images video stopped at iteration no. $=293$ and 201, and in 49 images video it stopped at iteration no. $=120$ and 162. In both videos, RMSE reached zero value at those iterations. In MLP, an error function plot is the result of a training phase process. From the above analysis we can conclude that increasing the number of input data sets makes the training process time faster.

\section{Table 1. MLP Tuning Parameters}

\begin{tabular}{|l|c|}
\hline Parameters & $\mathrm{e}^{\text {Valu }}$ \\
\hline Error goal & \multicolumn{2}{|c|}{ 1E- } \\
\hline Learning rate & 0.05 \\
\hline Momentum & 0.05 \\
\hline Iterations & \multicolumn{2}{|c|}{5000} \\
\hline Normalized SD & 0 \\
\hline Gradient vector & 0.4 \\
\hline
\end{tabular}

Table 2. Classification Results for 29 Images Video

\begin{tabular}{|c|c|c|c|c|c|c|c|}
\hline \multicolumn{4}{|c|}{ Subtraction method } & \multicolumn{4}{|c|}{ Features method } \\
\hline $\mathrm{ge}^{\text {Ima }}$ & $\mathrm{y}$ & $\mathrm{d}$ & Class & $\mathrm{ge}^{\text {Ima }}$ & $\mathrm{y}$ & d & Class \\
\hline $23^{\mathrm{img}}$ & $\begin{array}{c}- \\
0.9998\end{array}$ & 1 & breath in & $22^{i m g}$ & 1 & 1 & breath out \\
\hline $24^{i m g}$ & 1 & 1 & breath out & $23^{i m g}$ & 1 & 1 & breath out \\
\hline $25^{i m g}$ & -1 & $1^{-}$ & breath in & $24^{i m g}$ & $9^{0.999}$ & 1 & breath out \\
\hline $26^{i m g}$ & 1 & 1 & breath out & $25^{\text {img }}$ & -1 & 1 & breath in \\
\hline $27^{i m g}$ & $\begin{array}{c}- \\
0.7776 \\
\end{array}$ & $1^{-}$ & breath in & $26^{i m g}$ & 1 & 1 & breath out \\
\hline $28^{\text {img }}$ & -1 & 1 & breath in & $27^{\text {img }}$ & 1 & 1 & breath out \\
\hline & & & & $28^{i m g}$ & -1 & 1 & breath in \\
\hline
\end{tabular}

From the above factors we classified both videos, using them in different ways for defining the desired values. As mentioned before, the thermal image features have two sources to decide on the desired values. The first one uses a subtraction breathing plot to find out the breathing status and the second calculates the mode for each subtracted feature row. For example, if we have a different sign value for each feature in an image presented

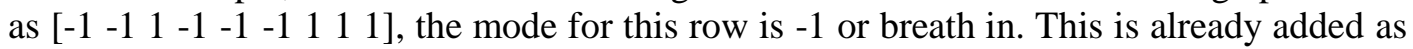
a loop in MLP code. The mode for features of each image will be the desired value of each one. In this step, we did not need to ignore any of the unclear images because we had numbers instead of plots, as in subtraction plot. 
The 29 image video was classified using different feature selection methods. The RMSE results for the 6 and 7 test images which used the subtraction plot as a desired values source are 9.917e-007 and 9.257816e-007 using 293 and 201 epochs respectively.

Table 3. Classification Results for $\mathbf{4 9}$ Images Video

\begin{tabular}{|c|c|c|c|c|c|c|c|}
\hline \multicolumn{4}{|c|}{ Subtraction method } & \multicolumn{4}{|c|}{ Features method } \\
\hline ge & $y$ & $d$ & Class & ge $e^{\text {Ima }}$ & $y$ & d & Class \\
\hline $33^{i m g}$ & -1 & $1^{-}$ & breath in & $31^{\mathrm{img}}$ & -1 & 1 & breath in \\
\hline $34^{i m g}$ & -1 & $1^{-}$ & breath in & $32^{i m g}$ & 1 & 1 & $\begin{array}{l}\text { breath } \\
\text { out }\end{array}$ \\
\hline $35^{\text {img }}$ & 1 & 1 & $\begin{array}{l}\text { breath } \\
\text { out }\end{array}$ & $33^{\mathrm{img}}$ & -1 & $1^{-}$ & breath in \\
\hline $36^{\text {img }}$ & -1 & $1^{-}$ & breath in & $34^{i m g}$ & -1 & & breath in \\
\hline $37^{i m g}$ & 1 & 1 & $\begin{array}{l}\text { breath } \\
\text { out }\end{array}$ & $3^{i m g}$ & -1 & $1^{-}$ & breath in \\
\hline $38^{i m g}$ & -1 & 1 & breath in & $36^{\text {img }}$ & -1 & $1^{-}$ & breath in \\
\hline $39^{\mathrm{img}}$ & 1 & 1 & $\begin{array}{l}\text { breath } \\
\text { out }\end{array}$ & $3^{i m g}$ & 1 & 1 & $\begin{array}{l}\text { breath } \\
\text { out }\end{array}$ \\
\hline $4^{\text {img }}$ & -1 & 1 & breath in & $3^{\text {img }}$ & $2^{0.926}$ & 1 & $\begin{array}{l}\text { breath } \\
\text { out }\end{array}$ \\
\hline $41^{i m g}$ & $\begin{array}{c}- \\
0.6691\end{array}$ & 1 & breath in & $3^{\text {img }}$ & 1 & 1 & $\begin{array}{l}\text { breath } \\
\text { out }\end{array}$ \\
\hline $42^{\text {img }}$ & 1 & 1 & $\begin{array}{l}\text { breath } \\
\text { out }\end{array}$ & $4^{\text {img }}$ & -1 & $1^{-}$ & breath in \\
\hline $4^{\text {img }}$ & 1 & 1 & $\begin{array}{l}\text { breath } \\
\text { out }\end{array}$ & $4^{\text {img }}$ & $\begin{array}{c}- \\
0.9986 \\
\end{array}$ & $1^{-}$ & breath in \\
\hline $4^{\text {img }}$ & $\begin{array}{c}- \\
0.9918 \\
\end{array}$ & & breath in & $42^{\text {img }}$ & 1 & 1 & $\begin{array}{l}\text { breath } \\
\text { out }\end{array}$ \\
\hline $4^{\text {img }}$ & 1 & 1 & $\begin{array}{l}\text { breath } \\
\text { out }\end{array}$ & $43^{\text {img }}$ & $\begin{array}{ll} & 0.581 \\
7 & \\
\end{array}$ & 1 & $\begin{array}{l}\text { breath } \\
\text { out }\end{array}$ \\
\hline $48^{\text {img }}$ & $1^{0.669}$ & 1 & $\begin{array}{l}\text { breath } \\
\text { out }\end{array}$ & $44^{i m g}$ & 1 & 1 & $\begin{array}{l}\text { breath } \\
\text { out }\end{array}$ \\
\hline & & & & $4^{i m g}$ & -1 & $1^{-}$ & breath in \\
\hline & & & & $46^{i m g}$ & 1 & 1 & $\begin{array}{l}\text { breath } \\
\text { out }\end{array}$ \\
\hline & & & & $4^{i m g}$ & $\begin{array}{c}- \\
0.9997\end{array}$ & $1^{-}$ & breath in \\
\hline & & & & $48^{i m g}$ & $\begin{array}{c}- \\
0.9986\end{array}$ & $1^{-}$ & breath in \\
\hline
\end{tabular}

\subsection{Calculating Breathing Rate}

The last step in the MLP classification system is to calculate the breathing rate for subjects. The breathing cycle is calculated every time the process is repeated. 
An example of breathing classification results is shown in Figure 6. For the 7 second video we got different result types. The breathing cycle was counted for every breath in the process we found during the results sequences. Here we had 2 cycles every 7 seconds, meaning the average breathing rate per 1 minute was 17 cycle/min. Table 4 summarizes all the breathing rates that were calculated for both perceptron and MLP systems.

The normal breathing rate range is $12-18$ cycles per minute for adults. Table 3 is within this range. This means that those under observation in the tests are breathing well. The results are quite perfect for dJetecting any abnormal breathing processes and so can help with making early diagnoses.

\section{Cycle $1 \quad$ Cycle 2 [IN OUT IN OUT OUT IN OUT]}

Figure 6. Example of Breathing Classification Results

Table 4. Classification System Breathing Rates

\begin{tabular}{|c|c|c|}
\hline $\begin{array}{c}\text { Tabl } \\
\text { e no. }\end{array}$ & $\begin{array}{c}\text { Cycle } / \text { Sec. calculated from } \\
\text { classification results }\end{array}$ & Breathing rate (avg BR per min) \\
\hline 5 & 2 cycles per 8 seconds & 15 cycles $/$ min \\
\hline 6 & 7 cycles per 18 seconds & 23 cycles $/$ min \\
\hline 7 & 2 cycles per 6 seconds & 20 cycles $/ \mathrm{min}$ \\
\hline 8 & 1 cycle per 7 seconds & 8 cycles $/ \mathrm{min}$ \\
\hline 9 & 4 cycle per 14 seconds & 17 cycles $/ \mathrm{min}$ \\
\hline 10 & 4 cycles per 18 seconds & 13 cycles $/ \mathrm{min}$ \\
\hline
\end{tabular}

\section{Conclusion and Future Work}

In this paper, we showed that a monitoring breathing system is able to include a neural network classification system as one of its tasks. Moreover, the calculation of the classified breathing cycles, represented as a breathing rate, can be another part of such a system. The supervised neural networks classifier can learn input data patterns and has the ability to test another group of input patterns. The MLP is capable of learning thermal image patterns as inputs and process them to produce the system output, by which the new image patterns are matched with their desired values in order to produce a breathing pattern with high accuracy.

In order to improve the classification results, especially for medical issues, patient (subject) results can be stored in a database. Each subject can have a private record and this record can be updated when the system receives more videos for the subject. The database records can be sent as data sheets using digital communication methods such as e-mail or via computer networks or mobile messages. For disease diagnosis, the records will enable consultants or researchers to remotely monitor their patients at all times.

\section{Acknowledgements}

Authors would like to thank Prof. Reza Saatchi and Dr. Farah Al-Khalidi for providing the required technical assistance and the data for this research project. 


\section{References}

[1] J. Fei and I. Pavlidis, "Analysis of breathing air flow patterns in thermal imaging”, EMBS '06. 28th Annual International Conference of the IEEE Engineering in Medicine and Biology Society, Aug (2006), pp. 946-952.

[2] R. Murthy and I. Pavlidis, "Non-contact monitoring of breathing function using infrared imaging," IEEE Engineering in Medicine and Biology Magazine, (2005).

[3] L. Tarassenko, L. Mason, and N. Townsend, "Multi-sensor fusion for robust computation of breathing rate," Electronics Letters, vol. 38, Oct (2002), pp. 1314-1316.

[4] J. Alihanka, Basic principles for analysing and scoring Bio-Matt (SCSB) recordings. Turunyliopisto, (1987).

[5] B. Larson, Signal Processing Techniques for Non-invasive Monitoring of Respiration and Heart Rate. University of Houston, (1987).

[6] R. Murthy, I. Pavlidis, and P. Tsiamyrtzis, “Touchless monitoring of breathing function,”. IEMBS '04. 26th Annual International Conference of the IEEE Engineering in Medicine and Biology Society, vol. 1, Sept (2004), pp. 1196-1199.

[7] E. Greneker, "Radar sensing of heartbeat and respiration at a distance with applications of the technology," in Radar 97, Conf. Publ. No. 449, Oct (1997) , pp. 150-154.

[8] J. Fei, Z. Zhu, and I. Pavlidis, "Imaging breathing rate in the co2absorption band," in. IEEE-EMBS 2005. 27th Annual International Conference of the Engineering in Medicine and Biology Society, (2005), pp. 700-705.

[9] S. Burnay, T. Williams, and C. Jones, Applications of thermal imaging. Adam Hilger, (1998).

[10] M. Frize, C. Herry, and R. Roberge, "Processing of thermal images to detect breast cancer: comparison with previous work," in Engineering in Medicine and Biology, 2002. 24th Annual Conference and the Annual Fall Meeting of the Biomedical Engineering Society EMBS/BMES Conference, 2002. Proceedings of the Second Joint, vol.2, (2002), pp. 1159-1160.Pavlidis, J. Levine, and P. Baukol, "Thermal imaging for anxiety detection," IEEE Workshop on Computer Vision Beyond the Visible Spectrum: Methods and Applications, (2000), pp. 104-109.

[11] I. Pavlidis and J. Levine, "Thermal image analysis for polygraph testing," Engineering in Medicine and Biology Magazine, IEEE, vol. 21, Nov (2002), pp. 56-64.

[12] J. A. Ware and I. Ciuca, "A neural network based integrated image processing environment for object recognition in medical applications", Tenth IEEE Symposium on Computer-Based Medical Systems, Jun (1997), pp. 149-154.

[13] M. Egmont-Petersen, D. de Ridder, and H. Handels, "Image processing with neural networks - a review," Pattern recognition, vol. 35, no. 10, (2002), pp. 2279-2301.

[14] M. Naka, T. Imai, T. Shida, M. Sato, R. Ito, and I. Akamine, "Thermal image processing using neural network," in IJCNN '93-Nagoya. Proceedings of 1993 International Joint Conference on Neural Networks, vol.3, Oct (1993), pp. 2065-2068.

[15] A.-K. Farah, R. Saatchi, H. Elphick, and D. Burke, "An evaluation of thermal imaging based respiration rate monitoring in children," American Journal of Engineering and Applied Sciences, vol. 4, no. 4, p. 586, (2012).

[16] F. Q. Al-Khalidi, R. Saatchi, D. Burke, and H. Elphick, "Tracking human face features in thermal images for respiration monitoring," in AICCSA, pp. 1-6, (2010).

[17] L. V. Fausett, Fundamentals of Neural Networks: Architectures, Algorithms, and Applications. Prentice Hall, (1994).

[18] P. Melin and O. Castillo, Modelling, Simulation and Control of Non-linear Dynamical Systems: An Intelligent Approach Using Soft Computing and Fractal Theory. CRC Press, (2001). 